\title{
NATURALLY DERIVED COMPOUNDS USED FOR PREVENTION OR REGRESSION OF EXPERIMENTALLY INDUCED LIVER FIBROSIS
}

\author{
Aya Adel* ${ }^{1}$, Mohe.M. Elmazar ${ }^{1}$, Amany A.E Ahmed ${ }^{2}$ 'Engy M. El Morsy ${ }^{2}$ \\ ${ }^{1}$ Center of Drug Research and Development, Faculty of Pharmacy, The British \\ University in Egypt, Cairo, Egypt \\ ${ }^{2}$ Department of Pharmacology and Toxicology, Faculty of Pharmacy, Helwan \\ University, Cairo, Egypt
}

*Corresponding author: aya.adel@gmail.com

\begin{abstract}
Liver fibrosis is a common health problem that is associated with mortality and morbidity worldwide. The inappropriate tissue repair of damaged liver results in oversynthesis and deposition of fibrillar collagen. It is usually associated with progressive pathological and biochemical changes that ultimately lead to structural and metabolic abnormalities and hepatic scarring. If not properly treated, liver fibrosis may develop to cirrhosis and hepatocellular carcinoma within few years. Blocking this progression, therefore, could be an effective and potential strategy for survival. The only effective approach to treating advanced liver fibrosis is transplantation. Understanding its etiology and pathophysiology, however, could help to investigate therapeutic pathophysiology based treatment of liver fibrogenesis. Recently several promising natural based treatment methods interfering with cytokines signaling pathway involved in fibrogenesis have been investigated offering new potential therapeutic intervention. The aim of the present updated review is to identify the natural antifibrotic options that have been studied in animal models with liver fibrosis for the treatment of this pathological conditions enabling prevention or at least regression of its progression.
\end{abstract}

Keywords: Liver Fibrosis, Cytokines Signaling Pathway, Antifibrotic, Cirrhosis Fibronectin, Elastin, and Laminin,

\section{Introduction}

Liver fibrosis is a critical public health concern with a worldwide mortality and morbidity attributable to cirrhosis and primary liver cancer that leads to 1.5 million deaths per year (WHO 2005). Fibrosis is defined as the wound healing response triggered by either acute or chronic stimuli that lead to accumulation of extracellular matrix. Hepatic fibrosis is the inappropriate tissue repair of the liver that results in oversynthesis and deposition of fibrillar collagen. It is usually associated with progressive pathological and biochemical changes that ultimately lead to structural and metabolic abnormalities and hepatic scarring (Mormone et al., 2011). Cirrhosis, on the other hand, is the progression or the end-stage consequence of liver fibrosis that is characterized by distortion of the normal architecture, altered blood flow, portal hypertension and finally hepatocellular carcinoma and permanent liver failure. Mild fibrosis remains mostly asymptomatic and may be reversible, however, its progression towards cirrhosis is generally irreversible and constitute the major cause of morbidity and mortality. It is urgent, therefore, to provide an effective antifibrotic agent in treating hepatic injury and 
fibrosis (Mormone et al., 2011). The most common etiological causes of liver fibrosis are, chronic viral hepatitis B and C, alcoholic liver diseases, and nonalcoholic fatty liver diseases, in addition to, parasitic infections, such as Schistosoma species (Poynard et al., 2003). The prevalence of liver fibrosis is predicted to increase due to the rising prevalence of obesity and metabolic syndrome, especially in developed countries (Lim and Kim 2008).

The treatment of choice of liver fibrosis is liver transplantation, however, immunological rejection, surgery- related complications, and the high costs associated with this procedure still constitute an obstacle to the transplantation (Elmahdy et al., 2017). For now, only reducing alcohol intake and the successful treatment of viral hepatitis could contribute in controlling the process.

Pathogenesis of liver fibrosis is complex and varies according to the different causes of hepatic injuries. After an acute liver damage, parenchymal cells regenerate and substitute the necrotic and apoptotic cells. Usually, this process is associated with an inflammatory response and a minor deposition of extracellular matrix (ECM) proteins in the extracellular space. If the injury persisted, eventually the regenerative response will fail and the hepatocytes will be replaced by abundant ECM, primarily composed of collagen type I-III-IV, fibronectin, elastin, laminin, and proteoglycans (Reeves and Friedman 2002). Excess ECM accumulation usually results in tissue scaring. It is generally accepted that activation of hepatic stellate cells (HSCs) is the central event in the process of fibrosis as it constitutes the major ECM- producing cells in the injured liver (Elsharkawy et al., 2005). (Fig. 1) Thus, the therapeutic efficacy of antifibrotic drugs can be improved by targeting HSCs.

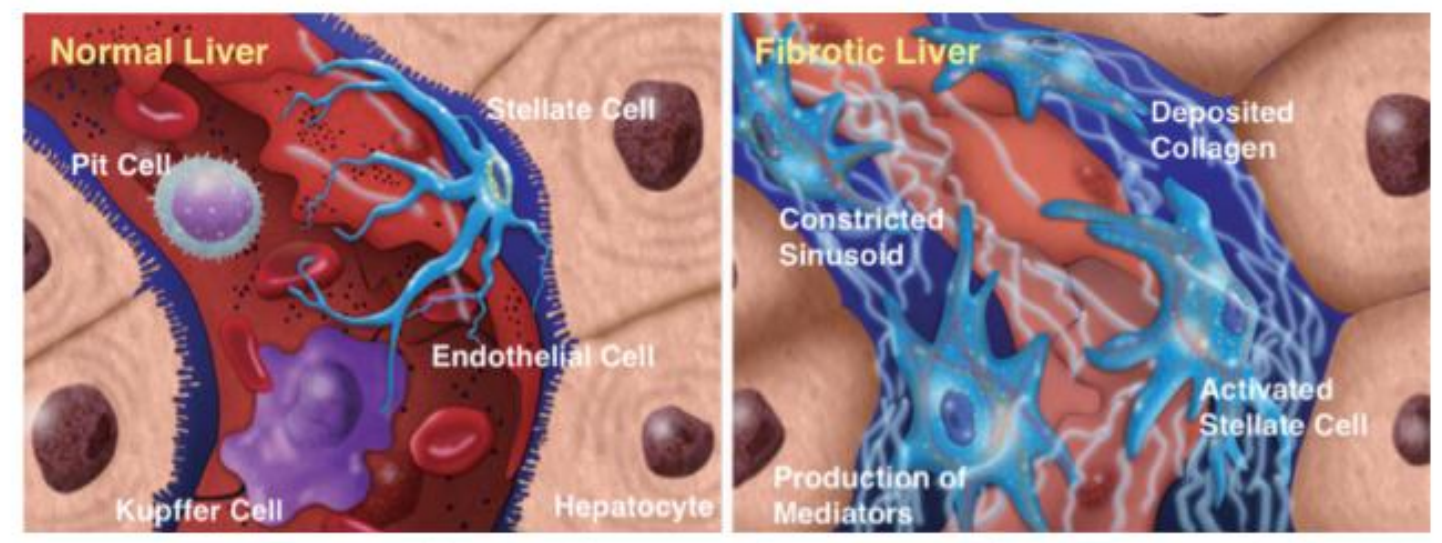

Fig 1: descriptive demonstration of normal and fibrotic liver. (Left) Hepatic sinusoid of intact liver is composed of endothelial cells with sieve plate, to which Kupffer cells and pit cells adhere. Stellate cells with vitamin A-droplets in the space of Disse attach to both endothelial cells and hepatocytes with their cytoplasmic processes. (Right) In fibrotic liver, stellate cells become activated and secrete type I collagen that is deposited in the space of Disse and profibrogenic and inflammatory mediators. Activated stellate cells constrict sinusoids, leading to microcirculatory disturbance in advanced fibrosis and ultimately to portal hypertension (Kawada 2011). 
Liver fibrosis may occur through different pathological and physiological scenarios. Inflammation is a critical and complicated feature of liver fibrosis. Liver injury is usually followed by an accumulation of recruited inflammatory cells in the injurious site (Duval et al., 2015). Different cell types of the innate or adaptive immune response participate in the fibrogenesis process such as, platelets, neutrophils, macrophages, mast cells and natural killer (NK) cell or T- and B cells. A wide repertoire of pro- and antiinflammatory compounds, including cytokines, chemokines, growth factors, and oxidative stress products, mediate the inflammatory response of immune cells during the fibrosis process (Duval et al., 2015). Kupffer cells is a major contributor to liver inflammation and they represent the resident liver macrophages. As a reflex to hepatic injury, kupffer cells get activated, expressing cytokines and signaling molecules.

Activated Kupffer cells express a dual /two marker of macrophages based on the signal they receive from the surrounding environment, M1-like macrophages (the inflammatory kupffer cells) or M2-like macrophages (the anti-inflammatory kupffer cells). Liver inflammation is regulated by the balance between M1 and M2 signals (Wan et al., 2014).

Oxidative injury is another common scenario that leads to liver fibrosis. It represents the disturbance in the oxidant-antioxidant balance resulting in a potential cellular damage. Normally, most cells are able to tolerate a mild degree of oxidative stress, because of the sufficient antioxidant defense capacity and repair systems that recognize and remove molecules damaged by oxidation. The imbalance occur when there is lack of antioxidant capacity due to a disturbance in production and distribution, or the overproduction of reactive oxygen species (ROS) from other factors (Ha et al., 2010). A biochemical stress is usually initiated by drugs and their reactive metabolites. The generated ROS directly affect HSCs and myofibroblast through activating signaling transduction pathways and transcription factors as Nuclear factor $\kappa \mathrm{B}(\mathrm{NF}-\kappa \mathrm{B})$, resulting in upregulation of the fibrotic associated genes, COL 1a1, COL 1a2, and tissue inhibitor of metalloproteinase 1 (TIMP1) (Ramon et al., 2005). Moreover, ROS decreases the antioxidant defense mechanisms such as glutathione (GSH) or Superoxide dismutase (SOD) along with enhanced lipid peroxidation leading to a profibrogenic response through enhancing collagen 1 protein expression (Mormone et al., 2011).

Three phases (i.e initiation, perpetuation and resolution) regulate the whole liver fibrosis process, the initiation phase usually represents the very early changes in the gene expression and phenotype. HSCs as an example, are stimulated by paracrine signals, such as, exposure to lipid peroxides and products released from damaged hepatocytes as well as biochemical signals from Kupffer and endothelial cells. In the perpetuation phase, the activated phenotype of HSCs is maintained, HSCs begin to lose their large vitamin A-containing lipid droplets and up-regulate the expression of cell adhesion molecules, and then fibrosis starts to generate. In the resolution phase, HSCs either undergo reversion to a quiescent phenotype or to clearance through apoptosis (Berardis et al., 2015).

The process following liver injury involves an acute and a chronic response. Once hepatic stellate cells are activated, and conversion from a quiescent to an activated state occurred, cytokine and chemokine are released. As a result they undergo transdifferentiation into myofibroblast-like cells and $\alpha$ smooth muscle actin ( $\alpha \mathrm{SMA}$ ) 
and procollagen-I starts to appear which further results in increased production and accumulation of extracellular matrix (ECM) components and collagen that are mainly regulated by two inflammatory mediators transforming growth factor-beta (TGF $\beta 1$ ) and tumor necrosis factor alpha (TNF $\alpha$ ) (Balta et al., 2015; Duval et al., 2015; Xu et al., 2012). TGF $\beta 1$ that is produced by Kupffer cells, endothelial cells and hepatocytes, is known to be the key activator of HSCs. It acts by activation of the Smad signaling pathway (Balta et al., 2015).

\section{Genes regulating liver fibrosis: Molecular mechanisms}

The response of hepatocytes to inflammation has a crucial decisive role in hepatic fibrosis. These cells will magnify the response throughout the production of other cytokines and chemokines, which increase the stimulus of hepatic stellate cells by activating pro-inflammatory cells (Fig.2). (Altamirano-Barrera et al,. 2017).

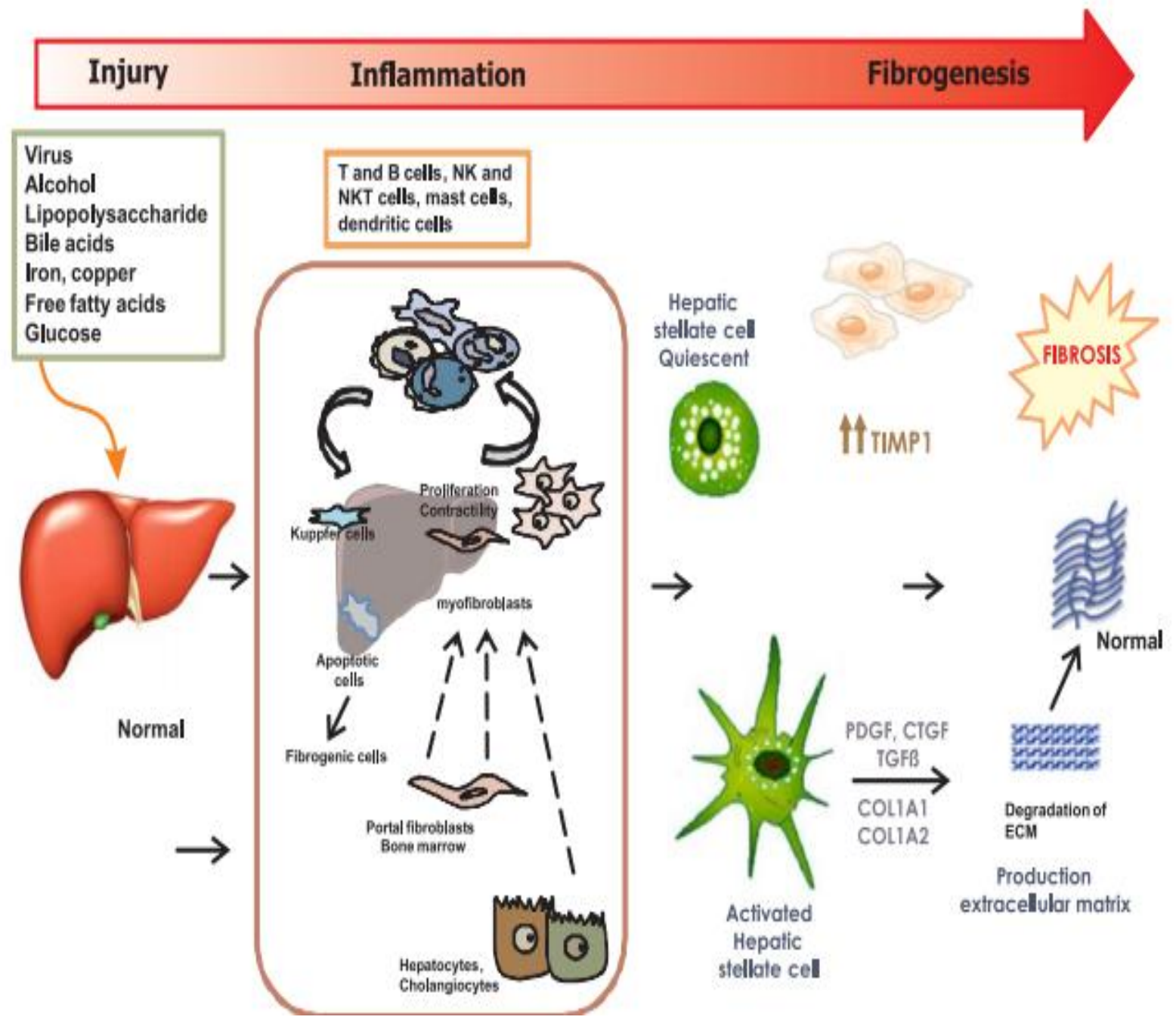

Fig 2: Mechanisms of hepatic fibrosis. Liver injury could be induced by several factors. These factors in turn results in liver inflammation through different types of cells. Hepatic stellate cells are the key cells in the process of liver fibrosis. NK cells: natural killer cells. PDGF: platelet-derived growth factor. CTGF: connective tissue growth factor. TGF $\beta$ : transforming growth factor- $\beta$. 
COL1A1: collagen type I alpha 1. COL1A2: collagen type I alpha 2 (Altamirano-Barrera et al., 2017).

Fibrogenesis is mediated throughout different cytokines and growth factors. TGF $\beta 1$ is one of the most potent fibrogenic cytokines. It has different isoforms and usually persist in an inactivated protein, and upon its activation, it sends signals through its receptors to the Smad proteins which in turn increase the transcription of the target fibrogenic genes like procollagens. (Inagaki and Okazaki 2007) It functions to activate HSCs, stimulate ECM synthesis and to decelerate their degradation (Yoshida and Matsuzaki 2012). Platelet derived growth factor (PDGF) is another potent mitogen for HSCs activation that is overexpressed in liver fibrosis and its inhibition has shown an alleviation in hepatic fibrosis of experimental animals (Pinzani et al., 1989). The vasoactive cytokine, Angiotensin II, also has a role in the progression of liver fibrosis as it triggers liver inflammation and induce a fibrogenic action on activated HSCs, such as cell proliferation and collagen synthesis (Bataller et al., 2003). Interleukin 6 (IL-6), also is one of the profibrotic cytokines that is produced by Kupffer cells and plays a role in the initiation and perpetuation of liver fibrosis (Peng et al., 2009). TNFa, a proinflammatory cytokine involved in the inflammatory and apoptosis response that also regulate NF- $\kappa \mathrm{B}$ (Latief and Ahmad 2018). It is produced by activated liver macrophages during acute inflammation caused by ROS exposure. It is responsible for the signaling events that serves in hepatocyte apoptosis and necrosis, immune cell activation and HSCs activation. Furthermore' it plays an important role in ECM remodeling, through induction of matrix metalloproteinase-9 (MMP9) and TIMP1 expression of HSCs (Seki and Schwabe 2015). NF- $\kappa \mathrm{B}$, is a transcription factor that is involved in the inflammation and apoptosis process. It plays a role in the activation of hepatocytes and HSCs and regulated the expression of many profibrotic cytokines. Its inhibition led to cell protection from apoptosis (Duval et al., 2014). $\alpha$ SMA constitutes a critical liver fibrosis factor as it helps in the detection of myofibroblast like cells as a reliable marker for the activated HSCs that are corresponding to late fibrosis stage (Tsai et al., 2008). Usually, the high $\alpha$ SMA expression level, directly correlates to the extent of disease progression (Berardis et al., 2015). In addition, Increased $\alpha$ SMA expression have been observed to be associated with the up regulation of various pro-fibrogenic genes, including procollagens $\alpha 1$ (I) and $\alpha 1$ (III), fibronectin, prolyl 4-hydroxylase that provide structural stability to collagens, TGF $\beta 1$, and TIMP1 (Lang et al., 2000). Collagen type I and III that are produced by the activated HSCs, are significantly upregulated during fibrosis as a result to the imbalance between the ECM degradation and production. Therefore, they constitute the major component of the scar matrix (Schuppan et al., 2001). matrix metalloproteinase (MMP) are generated mainly by the activated HSCs to degrade the fibrillar collagen of the ECM lying on the basement membrane then recruit the inflammatory cells to the scar site (Kisseleva and Brenner 2008). Oxidative stress also increases the expression of MMP 2 mRNA and stimulate the secretion of the active form of the enzyme (Knittel et al., 1999). Increased MMP2 expression and activity is believed to increase cell proliferation, migration and destruction of the normal liver architecture, thus, allowing for increased HSCs activation (Benyon and Arthur 2001). Connective Tissue Growth Factor (CTGF) is another critical hepatic fibrogenic factor. It is synthesized by both hepatocytes and HSCs. It constitutes a general mediator for the interaction between fiber and matrix in the injured tissue and plays a major role in increasing ECM-producing fibroblasts (Gressner and Gressner 2008). 
For these reasons, understanding these mechanisms and their modulation has its impact on developing new therapeutic strategies and options for preventing or regressing the process of liver fibrogenesis and improving hepatic function.

There are various animal models of liver fibrosis with different induction methods including administration of chemical hepatotoxins such as carbon tetrachloride $\left(\mathrm{CCl}_{4}\right)$, thioacetamide (TAA), dimethyl nitrosamine (DMN), alpha naphthyl isothiocyanate (ANIT) and diethoxycarobonyl dihydrocollidin (DDC). Mechanical induction could be performed by bile duct ligation (BDL) and immunological induction by the use of concanavalin-A besides the transgenic models. Animal modeling serve to elucidate the pathogenesis and to complement the different mechanisms of fibrosis as seen in humans in addition to explaining potential of different antifibrotic agents (Weiler-Normann et al., 2007).

\section{Natural prevention or mitigation of fibrotic progression}

Liver fibrosis progress to end up with irreversible condition of cirrhosis and ultimate liver failure or hepatocellular carcinoma if not managed properly. Therefore, the center of attention in treating liver fibrosis is the early stage of fibrosis. The Complementary and Alternative Medicine (CAM), is the new trend in handling chronic diseases as they possess a natural healing process and a long acting effects along with negligible side effects when experimentally evaluated (Latief and Ahmad 2018).

\section{Natural Herbal remedies}

\section{Anthocyanins}

Anthocyanins are flavonoids that are obtained from the fruits of Vaccinium oxycoccus, can also be extracted from various dietary supplements like grapes, peanuts and especially blueberry. It possesses biological and pharmacological properties such as hepatoprotective potential, antioxidant, anti-inflammatory and immunomodulatory effects (He and Giusti 2010). Sun et al. (2018) discussed the antifibrotic activity of anthocyanins which is referred to the suppression of HSCs activation in addition to decreasing oxidative stress and associated inflammatory responses post- $\mathrm{CCl}_{4}$ mediated liver fibrosis. Most importantly, anthocyanins had the ability to increase MMP9 and decrease TIMP1 level to modulate ECM remodeling cell. The mechanism of anthocyanins may extend to be related to reduction of ROS generating sources, decreasing the influence of pro-inflammatory cytokines, and downregulation of Col-III and $\alpha$ SMA (Sun et al., 2018).

\section{Armepavine}

Armepavine is an active alkaloid compound derived from plant Nelumbo nucifera. It possesses anti-inflammatory and immunosuppressive effects (Liu et al., 2006). It exerted an antifibrotic effect by downregulating the expression of TNF $\alpha$ and aSMA in TAA-induced liver fibrosis model in rat (Weng et al., 2012). Similar studies with different settings reported the antifibrotic effect of armepavine. In a BDL-induced liver fibrosis model, armepavine showed significant antifibrotic effect through lowering hepatic aSMA expression and collagen contents, fibrosis score and attenuated mRNA expression levels of Col 1a2, TGF $\beta 1$, TIMP1and IL-6 (Weng et al., 2009). 


\section{Baicalin}

Scutellaria baicalensis Georgi is one of the critical medicinal herbs used for treatment of cancer, viral diseases and inflammatory diseases in East Asian countries. It contains a large number of flavonoids, among them the flavone baicalin (5,6-dihydroxy4-oxygen-2- phenyl-4H-1-benzopyran-7-beta-D-glucopyranose acid). Baicalin exerts the inhibitory effects against several viruses including influenza virus, human $\mathrm{T}$ cell leukemia virus and acquired human immunodeficiency virus type (Zeng et al., 2007). It possess several pharmacological activities as potent anti-inflammatory, anti-allergic and antibacterial agent. It has significant scavenging effects on oxygen free radicals and protective effects on liver injury (Peng et al., 2009). In $\mathrm{CCl}_{4}$-induced hepatic fibrosis model in rat, baicalin lowered the levels of serum ALT, AST and liver index, decreased histological changes of liver fibrosis, inhibited the expression of cytokines, including TGF $\beta 1$, TNF $\alpha$ and IL-6, and improved the serum level of IL-10 significantly (Peng et al., 2009). Further study showed that the increases of several fibrosis indices including serum hyaluronic acid, collagen and hepatic hydroxyproline content after $\mathrm{CCl}_{4}$ injection can be significantly inhibited by baicalin treatment (Li et al., 2008). Baicalin has significant antifibrogenic effects of liver fibrosis in rats not only by the inhibition of HSCs activation and lipid peroxidation but mainly by the immune-regulation of the imbalance between profibrotic and antifibrotic cytokines (Peng et al., 2009).

\section{Chrysin}

Chrysin (5,7-dihydroxyflavone) is a flavonoid, extracted from honey, propolis and many plants. The medicinal value of chrysin is due to its anti-inflammatory, anticancer, antithrombotic, anti-allergic and antioxidant activities (Farkhondeh et al., 2015). Chrysin down-regulation of Smad 2 and 3, which inhibits TGF $\beta /$ Smad signaling may be the key antifibrotic mechanism that attenuate $\mathrm{CCl}_{4}$-induced liver fibrosis (Balta et al., 2015). The Smad signaling pathway is important for transmitting TGF $\beta 1$ signals from the cell surface to the nucleus. Moreover TGF $\beta 1$ exerts its fibrogenic effect through phosphorylation of Smad 2 and 3(Weiss and Attisano 2013). In addition Chrysin has been demonstrated to reduce the number of activated HSCs, as proved by the reduced myofibroblast marker $\alpha$ SMA expression (Balta et al., 2015). The ability of chrysin to suppress TIMP1, MMP2, MMP3, and MMP9 expressions and decreasing collagen deposition explain its antifibrogenic effect of liver fibrosis model in mice (Balta et al., 2018).

\section{Curcumin derivative C66}

Turmeric has been used as a traditional medicine in many countries, curcumin, the principal curcuminoid of turmeric, has been reported as the biological active component responsible for most of its activities. Curcumin has been known to be a promising antitumor, antioxidant, anti-inflammatory and antifibrogenic agent (Tang 2015). It is believed that curcumin exerts its function by suppressing HSCs activation through different signaling pathways such as Wnt/ $\beta$-catenin (Cui et al., 2014), TGF $\beta /$ Smad (Yao et al., 2012) and Shh signaling pathways (Qiu et al., 2014) and by inhibiting HSCs angiogenesis via PDGF- $\beta$ R/FAK/RhoA signaling pathway (Zhang et al., 2014). Curcumin uses were limited due to its poor solubility so Huang et al. (2016) have developed a new curcumin derivative (2E,6E)2,6-bis (2 (trifluoromethyl) benzylidene) cyclohexanone (C66) and studied its antifibrotic activity against $\mathrm{CCl}_{4}$ - 
induced liver fibrosis model in mice. The results clearly demonstrated that C66 has more anti-inflammatory and antifibrotic effect than curcumin itself. It reduced hepatic collagen-I, collagen-III contents and hepatic hydroxyproline concentration along with improving the organizational architecture damaged by $\mathrm{CCl}_{4}$ and countervailing the increase in IL-6, TNF $\alpha$, and TGF $\beta 1$. More importantly, the effect of C66 in attenuating liver fibrosis is likely through a mechanism involving cannabinoid receptor and $\mathrm{JNK} / \mathrm{NF}-\kappa \mathrm{B}$ activation (Huang et al., 2016).

\section{Dioscin}

Dioscin, a plant steroid saponin, is widely prevalent in many herbs (Dong et al., 2012). It possesses anti-inflammatory, lipid-lowering and anticancer activities (Lu et al., 2011). Several investigations have shown that dioscin is highly effective against liver injury and non-alcoholic fatty liver diseases (Zhang et al., 2015a). Zhang et al. (2015b) have related the antifibrotic potential of dioscin with the inhibition of HSCs activation regulating the balance between collagen synthesis and degradation, which leads to decrease in the accumulation of ECM and the inhibition of TGF $\beta /$ Smad and P38 MAPK signaling pathways. In addition, dioscin was also shown to suppress oxidative stress, inflammatory markers as, IL-1 $\beta$, IL-6 and TNF $\alpha$ expression in TAA-induced liver fibrosis model (Zhang et al., 2015b). Dioscin decreased the typical HSCs activation markers, laminin, Col $1 \mathrm{a} 1$ and $\mathrm{Col} 3 \mathrm{a} 1$ in $\mathrm{CCl}_{4}$-induced model of liver fibrosis suggesting that the mechanism of dioscin is mediated through induction of apoptosis in the activated HSCs. Additionally dioscin suppressed pro-inflammatory cytokine production, NF- $\kappa \mathrm{B}$ activation and downregulated inflammatory cytokines including TNF $\alpha$, IL-1 $\beta$, IL-6, and Cyclooxygenases 2 (COX-2) indicating that the antifibrotic effect of dioscin may be mediated through inflammation suppression (Zhang et al., 2015a).

\section{Garlic extracts (GE)}

Garlic (Allium sativum L. Amaryllidaceae) has been consumed as a flavoring agent and a traditional medicine in treating tuberculosis, coughs, colds and hyperpiesia (Wang et al., 2018). Few years ago, it has been found in certain models, that garlic preparations prevented tumor progression, cardiovascular and liver diseases and aging that was associated with its scavenging effect of oxygen radical and lipid peroxidation (Gedik et al., 2005). GE elicited therapeutic effect against $\mathrm{CCl}_{4}$-induced liver fibrosis. Regression of liver fibrosis occurred by reducing myofibroblasts via modulation of HSCs activation mechanisms, ECM remodeling by stimulating its degradation and regenerating liver tissue and functions (D'Argenio et al., 2013). Moreover, the aqueous extract of garlic had a key role in inhibiting biliary obstruction induced liver fibrosis and oxidative damage with its potent free radical scavenging and antioxidant properties. Its action extends to reduce TNF $\alpha$, TGF $\beta 1$, MMP13, myeloperoxidase, collagen content and increasing GSH levels (Gedik et al., 2005; Mahmoud et al., 2014).

\section{Glycyrrhizin and Glycyrrhizic acid}

Licorice is the root of Glycyrrhiza Glabra. Licorice, is rich in secondary metabolites that are associated with numerous pharmacological activities. It has been reported to have useful effects against various diseases like tuberculosis, atherosclerosis, bacterial infection and cancer (Sidhu et al., 2018). The licorice flavonoids prevented 
TAA-induced liver fibrosis in rats by down-regulation of TGF $\beta 1$ and Caspase- 3 protein expressions (Jing et al., 2015).

Glycyrrhizin (GL) is the main source of the sweetness of licorice. It has 2 isomers, $18 \alpha-\mathrm{GL}$ and $18 \beta-\mathrm{GL}$. $18 \alpha-\mathrm{GL}$ have been tested against $\mathrm{CCl}_{4}$-induced liver fibrosis, and the results showed that it possesses antifibrogenic properties and improved liver fibrosis by acting on TGF $\beta /$ Smad signal pathway (Qu et al., 2015).

Glycyrrhizic acid (GA), is a major active component of licorice. It has been reported to have anti-inflammatory, antiviral, anti-apoptotic and hepatoprotective activities (Wang et al., 2016). GA was reported to reduce liver fibrosis through limiting HSCs activation and collagen deposition (Wang et al., 2016). Liang et al. (2015) discussed the effect of GA against liver fibrosis induced by $\mathrm{CCl}_{4}$. GA treatment ameliorated fibrosisrelated factors and exerted a beneficial anti-apoptotic effect on activated hepatocytes. Moreover, treatment with GA significantly enhanced the pathological changes in the fibrotic liver, inhibited hepatic stellate cell activation and reduced expression of caspase-3, Bax, aSMA, CTGF, MMP2 and MMP9 proteins, and aSMA mRNA, collagen type I and III (Liang et al., 2015; Çetin et al., 2016).

\section{Hesperidin (HDN)}

Hesperidin (HDN) is a bioflavonoid that is abundant in citrus fruits. It has an effective antioxidant, anti-inflammatory, free radical scavenging properties, anticarcinogenic, and antihypertensive agent with lipid-lowering activities. (Çetin et al., 2016). They assured that a dose of $50 \mathrm{mg} / \mathrm{kg} /$ day HDN for 10 consecutive days reversed the oxidative effects of $\mathrm{CCl}_{4}$ via a significant reduction of thiobarbituric reactive substances (TBARS) levels and stimulating the antioxidant defense system. Further study has confirmed these results as Bentli et al. (2013) who determined that HDN protected the liver against dioxin toxicity and prevented liver injury. In addition, Chen et al. (2010) determined that HDN reduced oxidative stress indicators, like ROS and lipid peroxidation, in a dose-dependent manner. Suppressing aSMA expression via inhibition of HSCs activation, inhibition of caspase-dependent apoptosis, restoration of diminished GSH content, reduction of the elevated malondialdehyde (MDA) content and reduction of iNOS expression after DMN-induced fibrosis in rats are all attributed to the antioxidant and anti-apoptotic effects of HDN (Elshazly and Mahmoud 2014). In $\mathrm{CCl}_{4}$-induced liver fibrosis model, $\mathrm{HDN}$ showed significant antifibrotic mechanism associated with its ability to reduce oxidative stress and modulate proinflammatory and profibrotic signals. It modulated the effects of $\mathrm{CCl}_{4}$ toxicity, inhibited $\mathrm{NF}-\kappa \mathrm{B}$ and decreased the expression of TGF $\beta 1$, CTGF and IL-1 $\beta$ and upregulated IL-10 levels (Pérez-Vargas et al., 2014).

\section{Quercetin}

Quercetin (3,3,4,5,7-pentahydroxyfavone), is a flavonoid present in fruits and some vegetables like red onions and kale. It has a wide safety profile and bioavailability (Ferry et al., 1996). Quercetin possess an anti-inflammatory, antioxidant and antitumor activity, and has the ability to decrease the risk of chronic health conditions including cardiovascular, neurodegenerative diseases and diabetes (Harwood et al., 2007). Quercetin attenuated $\mathrm{BDL}$ or $\mathrm{CCl}_{4}$-induced hepatic fibrosis by inhibiting HSCs activation. Furthermore, it significantly exerted an antifibrotic activity by suppressing the TGF $\beta 1 /$ Smads signaling pathway and activating the PI3K/Akt signaling pathway 
(Wu et al., 2017). Wang et al. (2017) have reported that the antifibrotic mechanisms of quercetin against $\mathrm{CCl}_{4}$-induced liver fibrosis might be related to its ability to regulate $\mathrm{NF}-\kappa \mathrm{B} / \mathrm{I} \kappa \mathrm{B} \alpha, \mathrm{p} 38$ MAPK anti-inflammation signaling pathways to prevent inflammation, and regulate $\mathrm{Bcl}-2 / \mathrm{Bax}$ anti-apoptosis signaling pathway to inhibit liver cell apoptosis. Different hypothesis of the antihepatic fibrosis effect of quercetin might go back to its ability to regulate inflammation factors and HSCs activation markers, such as TNF $\alpha$, IL-6, IL-1 $\beta$, COX-2, TGF $\beta 1, \alpha$ SMA, Col la1, Col 1a2, TIMP1, MMP1, and desmin in the same fibrosis model (Wang et al., 2017).

\section{Resveratrol (RSV)}

(3,5,4'-trihydroxystilbene) is a natural polyphenol compound that is present in various plants, such as peanuts (Arachis hypogea), blue- and cranberries (Vaccinum spp), and grape vines (Vitis vinifera). It is considered to have broad spectrum biological activities including, antioxidant, anticarcinogenesis, anti-inflammatory, anti-aging and antimicrobial properties (Vestergaard and Ingmer 2019). Resveratrol regulated TAAinduced liver injury through caspase- 3 activation and inhibition of NF- $\kappa$ B and CYP2E1, which helps in the suppression of TAA biotransformation to its toxic metabolite (Ahmad and Ahmad 2014). Kessoku et al. (2016) demonstrated that RSV inhibited fibrosis and inflammation in a mouse model of nonalcoholic steatohepatitis (NASH) by inhibiting the activation of STAT3 pathway. DMN-induced liver fibrosis model in rats, oral administration of resveratrol was able to keep the damage to near normal biomarker levels, reserve histological integrity and block the progression of liver fibrosis. Moreover, resveratrol showed a significant antiproliferative, antifibrotic as well as antihypertensive activity (Abdu and Al-Bogami 2017).

\section{Sauchinone}

Sauchinone is a biologically active lignan found in Saururus chinensis, and is used as a traditional oriental medicine for treatment of fever, edema, jaundice and several inflammatory diseases. It has been reported to have hepatoprotective, antiinflammatory, and antioxidant effects (Sung and Kim 2000). It is reported to possess an antifibrotic activity by decreasing regions of hepatic degeneration, inhibiting inflammatory cell infiltration, and lowering the intensity of $\alpha$ SMA staining in mice model of liver fibrosis (Lee et al., 2014).

\section{Silymarin}

Silymarin is a herbal product extracted from the seeds of milk thistle plant (Silybum marianum) and contains at least three flavonolignans (silybin, silychristin, and silydianin). It was introduced as a hepatoprotective agent few years ago (Tsai et al., 2008). It offers hepatoprotection in various toxic models of experimental liver diseases in vivo through its free radicals scavenging properties, enhancing the endogenous antioxidant defense systems and stabilizing effect on the cytoplasmic membranes (Sokar et al., 2017). In addition, it stimulates protein synthesis, glucuronidation, protects against glutathione depletion and plays role in the regeneration of liver tissue. Furthermore, it possess a robust immunomodulatory, anti-inflammatory and antifibrogenic agent by its action on alteration of hepatic Kupffer cell function, lipid peroxidation, and collagen production (Jeong et al., 2005). Tsai et al. (2008) have stated that silymarin significantly reversed the altered expressions of $\alpha \mathrm{SMA}$ in $\mathrm{CCl}_{4}$-induced fibrotic liver tissue which was accompanied by a decrease in activated hepatic stellate 
cells number that will eventually lead to their apoptosis and resolution of liver fibrosis. Jeong et al. (2005) examined the inhibitory mechanism of silymarin on $\mathrm{CCl}_{4}$-induced hepatic cirrhosis in rats. They reported that the antifibrotic and anti-inflammatory effects of silymarin were associated with activation of HSCs through the expression of TGF $\beta 1$ and stabilization of mast cells. Mast cells are reported to be over-expressed in chronic liver diseases associated with liver fibrosis, and have a potential role in fibroblast growth and stimulating the production of the ECM by HSCs (Sugihara et al., 1999). In Jeong et al. (2005) study, the number of mast cells in portal areas was gradually increased in the fibrogenic stage, however, the number of mast cells in the $\mathrm{CCl}_{4} /$ silymarin-treated group was decreased significantly compared to those of $\mathrm{CCl}_{4}$ treated group.

\section{Cell based therapy}

Recently, stem cell treatment, commonly known as biologicals, has been suggested as an effective therapy for hepatic disease. The use of stem cells of different sources, including hematopoietic stem cells, mesenchymal stem cells, and umbilical stem cells might be a proactive option for treating chronic liver failure. Its mechanism of action is mainly based on four theories: either the ability of the cells to improve the hepatic inflammatory microenvironment, inhibition of the activation or inducing apoptosis of HSCs, replacing damaged hepatocytes and/or promoting the regeneration of residual hepatocytes (Berardis et al., 2015).

Bone marrow cells (BMCs) and Umbilical cord blood mononuclear cells (UCB MNCs) as examples that produce a series of growth factors and cytokines that can significantly downregulate inflammatory responses, suppress hepatic fibrosis and activate the proliferation of hepatocytes (Zhou et al., 2009).

Mesenchymal stem cells (MSCs) are adult stem cells that are found in the mesenchymal and connective tissues of various origins. The bone marrow, adipose tissue, placenta, umbilical cord and cord blood are few examples. These cells have an immunomodulation, self-renewal and multidirectional differentiation properties (Lin et al., 2011). MSCs have the capability to differentiate into multiple cell lineages including hepatocyte-like cells that perform hepatic functions such as albumin production, glycogen storage, urea secretion, low-density lipoprotein uptake and phenobarbitalinducible cytochrome P450 activity (Jiang et al., 2002). MSCs produce modulatory cytokines that help in inhibiting immunocyte proliferation and migration to the liver, thereby attenuating inflammatory injury, reducing hepatocyte apoptosis, and regressing liver fibrosis (Jiang et al., 2002; Lin et al., 2011). Adipose derived mesenchymal stem cells, (ADSCs) have the ability to inhibit liver fibrogenesis and promote apoptosis of HSCs in $\mathrm{CCl}_{4}$-induced liver fibrosis rat model (Yu et al., 2015).

The BMCs are considered to be the most promising source for the cell therapy in various diseases owing to its developmental plasticity (Oertel and Shafritz 2008). Li et al. (2013) studied the administration of murine bone marrow derived mesenchymal stem cells in $\mathrm{CCl}_{4}$-induced fibrosis model, and the results proved that MSCs have a great homing capability to the injured liver, a great ability to promote hepatocyte proliferation, inhibit hepatic stellate cell activation and facilitate mice survival. Furthermore, they assured the ability of these cells to attenuate chemically induced inflammation in the fibrotic liver (Li et al., 2013). Further studies have confirmed the 
same results regarding BMCs significant antifibrotic effect in reversing liver fibrosis as evidenced by the reduction in liver collagen, lipid peroxidation, increase in MMP13 and the downregulation of $\alpha$ SMA and TIMP1 (Rabani et al., 2010).

The placenta and umbilical cord blood (UCB) were used to be discarded after delivery as a medical waste. Recent studies have discussed the successful use of human umbilical cord blood stem cells in organs damage in vivo without the need for immune suppression (Chen et al., 2015). Tanabe et al. (2004), stated that UCB is a possible source of hematopoietic cells, which are stem cells that give rise to other blood cells, and could be delivered to the liver by the portal or systemic circulations and accordingly, can be used in treatment of hepatic failure. It has been found that Human UCB contains transplantable hepatic progenitor cells which are undoubtedly important for liver regeneration in severely injured liver (Tanabe et al., 2004). Since hepatocytes are the major contributors to liver regeneration, they however cannot proliferate. On the other hand, hepatic progenitor cells, called oval cells, invade into the adjacent liver parenchyma, and differentiate into hepatocytes and cholangiocytes (Shiota et al., 2000). These oval cells express receptors for different growth factors like, transforming growth factor K (TGFK), TGFL, and hepatocyte growth factor (HGF) (Shiota et al., 2000). Elmahdy et al. (2017) and Jung et al. (2009) have studied the effects of UCB MNC against $\mathrm{CCl}_{4}$-induced liver fibrosis model, and found out that profibrogenic marker TGF $\beta 1$, inflammatory marker TNF $\alpha$, hydroxyproline, laminin, and $\alpha$ SMA were significantly decreased compared to the $\mathrm{CCl}_{4}$ group, accordingly it represents a potential antifibrotic treatment in experimentally induced liver fibrosis.

Further study to confirm these findings against cirrhotic rat liver proved the differentiation of human UCB into hepatocyte like cells expressing the hepatocytespecific markers, human albumin and alpha-fetoprotein. In addition to significant inhibition of TGF $\beta 1$, collagen type I and $\alpha$ SMA expressions (Jung et al., 2009).

\section{Combined treatment for liver fibrosis}

\section{Diethylcarbamazine combined with Hesperidin}

Diethylcarbamazine (DEC) has been used in the treatment of human lymphatic filariasis. DEC has also anti-inflammatory properties as a result of its interference with arachidonic acid metabolism, which includes lipoxygenases (LOX) and COX enzymes. It blocks endothelial cell production of the cyclooxygenase pathway products PGE2, PGI2 and thromboxane A2. It is also described as a 5-lipoxygenase inhibitor, blocking the production of leukotrienes (Maizels and Denham 1992). Additionally, it affects different enzyme systems like folate metabolism, glycolysis and the activity of acetylcholinesterase (Subrahmanyam 1987). Rocha et al. (2012) revealed a clinical potential of DEC for therapeutic anti-inflammatory applications, as it inhibited the ethanol-induced hepatic injury and decreased inflammatory markers like NF- $\kappa \mathrm{B}$. According to Rocha et al. (2014) study, DEC has antifibrotic and anti-inflammatory effects through reducing the impact of proliferation and activation of HSCs by the inhibition of TGF $\beta 1$, and $\alpha$ SMA expression and reduction in COX-2, IL- $1 \beta$, and MDA in chronic $\mathrm{CCl}_{4}$ model of liver toxicity.

El-Sisi et al. (2017) elucidated that DEC when combined with hesperidin could strongly resolve liver fibrosis in ethanol-induced model in rats. The combination resulted in reduction to the serum levels of ALT, AST close to the normal levels, 
reduction in hepatic MDA, NO and elevation of GSH levels proving the synergistic effect of both drugs as antioxidants and antinitrosative agents. DEC plus hesperidin treatments showed significant suppression in the release of IL-6 possibly by inhibiting $\mathrm{NF}-\kappa \mathrm{B}$ activation more than each drug alone assuring the synergistic anti-inflammatory effect of the combination. The inhibition of TGF $\beta 1$ and $\alpha$ SMA expression and consequently reducing the impact of the proliferation and activation of HSCs confirmed the synergistic antifibrotic effect of both drugs.

\section{Human umbilical cord mononuclear cells combined with Silymarin}

Elmahdy et al. (2017) demonstrated the potential antifibrotic effect of human UCB MNC and silymarin in an experimental model of chronic $\mathrm{CCl}_{4}$-induced liver fibrosis in mice through investigating the effects on the profibrotic marker, inflammatory markers in addition to the oxidative and fibrotic markers. The combination showed a significant decrease in MDA, collagen, laminin, TGF $\beta 1$, TNF $\alpha$ and a significant increase in the GSH levels, in addition to a similar regression in the histological parameters.

\section{Mesenchymal stem cells and Interleukin-6}

The IL- 6 is one of the inflammatory cytokines produced by the liver in response to injury. A lot of evidences show that IL-6 can enhance hepatocyte survival while IL-6 depleted mice have showed liver fibrosis (Nasir et al., 2013). Exogenous IL-6 treatment correct the defects in cell proliferation in IL-6-/- mice proving its role as mitogenic agent in liver regeneration after partial hepatectomy (Sudo et al., 2008). Nasir et al. (2013) demonstrated that IL-6 administration significantly enhanced the ability of MSCs to restore liver condition after $\mathrm{CCl}_{4}$-induced fibrosis. The combination resulted in increased MSC engraftment leading to improved hepatic function. Furthermore, liver section stained with Sirius red for fibrosis and TUNEL staining for apoptosis detection showed a significant reduction in the combination treated group when compared with $\mathrm{CCl}_{4}$ group or each treatment alone (Nasir et al., 2013).

\section{Sitagliptin combined with Silymarin}

Sitagliptin, a dipeptidyl peptidase 4 (DPP4) inhibitor, has been known as a possible treatment for type-2 diabetes mellitus (DM), as it enhances the function of incretin by inhibiting DPP4, which causes degradation of the incretin hormones, glucose-dependent insulinotropic polypeptide (GIP) and glucagon-like peptide-1 (GLP1). Both GIP and GLP-1 inhibit glucagon secretion and stimulate insulin secretion from the $\beta$-cells. Therefore, the continuous inhibition caused by Sitagliptin to DPP4 lead to a higher bioavailability of these hormones and there upon lower serum glucose level. DPP4 is also highly expressed in liver, and plays a role in the fibronectin-mediated interaction of hepatocytes with ECM and is expressed on the surface of activated HSCs. Moreover, it was reported that its serum activity and mRNA levels were significantly increased in tetrachloromethane-induced cirrhotic rats and in nonalcoholic fatty liver disease (NAFLD) compared to that in control livers (Sokar et al., 2017).

Sitagliptin and silymarin combination resulted in more antifibrotic, antiinflammatory and antioxidant effects compared to single drug alone against $\mathrm{CCl}_{4^{-}}$ induced liver fibrosis. The effect was found to be mainly through the inhibition of hepatic oxidative stress and augmentation of anti-oxidant defenses, attenuating the 
activation of hepatic stellate cells via reduction of $\alpha$ SMA expression in liver, inhibiting the fibrogenesis and proliferation of activated HSCs response via inhibiting the release of TGF $\beta 1$, and inhibition of proinflammatory cytokines as IL-6. Interestingly, this combination may be useful for patients with type 2 Diabetes Mellitus accompanied by liver injury (Sokar et al., 2017).

\section{Conclusion}

Liver fibrosis is well known to be irreversible and progressive. However, a bundle of clinical studies proved that it can be reversed if handled effectively on the right time or if the causative agents have been withdrawn (Hammel et al., 2001). So far, there is no specific and effective antifibrotic therapy on record, but liver transplantation will remain the definitive treatment option for end-stage liver disease. The imbalance between the number of patients needing transplantation and the amount of available donors/organs will remain to be an obstacle highlighting the need to develop new therapeutic option to reduce liver scarring and stimulate liver regeneration. For these reasons, further research should focus on herbal medicine that are claimed to possess optimistic antifibrotic properties.

\section{REFERENCES}

Abdu SB, Al-Bogami FM (2017). Influence of resveratrol on liver fibrosis induced by dimethylnitrosamine in male rats. Saudi Journal of Biological Sciences 26:201-209

Ahmad A, Ahmad R (2014). Resveratrol mitigate structural changes and hepatic stellate cell activation in $\mathrm{N}^{\prime}$-nitrosodimethylamine-induced liver fibrosis via restraining oxidative damage. Chemico-Biological Interactions 221:1-12.

Altamirano-Barrera A, Barranco-Fragoso B, Méndez-Sánchez N (2017). Management strategies for liver fibrosis. Annals of Hepatology 16(1):48-56.

Balta C, Ciceu A, Herman H, Rosu M, Boldura OM, Hermenean A (2018). Dosedependent antifibrotic effect of chrysin on regression of liver fibrosis: the role in extracellular matrix remodeling. Dose-Response 16(3):155932581878983.

Balta C, Herman H, Boldura OM, Gasca I, Rosu M, Ardelean A, Hermenean A (2015). Chrysin attenuates liver fibrosis and hepatic stellate cell activation through TGF- $\beta /$ Smad signaling pathway. Chemico-Biological Interactions 240:94-101.

Bataller R, Gabele E, Schoonhoven R, Morris T, Lehnert M, Yang L, Brenner DA, Rippe RA (2003). Prolonged infusion of angiotensin II into normal rats induces stellate cell activation and proinflammatory events in liver. American Journal of Physiology-Gastrointestinal and Liver Physiology 285(3):G642G651.

Bentli R, Ciftci O, Cetin A, Unlu M, Basak N, Çay M (2013). Oral administration of hesperidin, a citrus flavonone, in rats counteracts the oxidative stress, the inflammatory cytokine production, and the hepatotoxicity induced by the ingestion of 2, 3, 7, 8-tetrachlorodibenzo-p-dioxin (TCDD). European Cytokine Network 24(2):91-96.

Benyon RC, Arthur MJ (2001). Extracellular matrix degradation and the role of hepatic stellate cells. In: Seminars in liver disease,. vol 21. by Thieme Medical Publishers,New York pp 373-384. 
Berardis S, Sattwika PD, Najimi M, Sokal EM (2015). Use of mesenchymal stem cells to treat liver fibrosis: current situation and future prospects. World Journal of Gastroenterology: WJG 21(3):742-758.

Çetin A, Çiftçi O, Otlu A (2016). Protective effect of hesperidin on oxidative and histological liver damage following carbon tetrachloride administration in Wistar rats. Archives of medical science: 12(3):486-493.

Chen M, Gu H, Ye Y, Lin B, Sun L, Deng W, Zhang J, Liu J (2010). Protective effects of hesperidin against oxidative stress of tert-butyl hydroperoxide in human hepatocytes. Food and Chemical Toxicology 48(10):2980-2987.

Chen Y, Ye L, Zhong J, Li X, Yan C, Chandler MP, Calvin S, Xiao F, Negia M, Low WC, Zhang J, Yu X (2015). The structural basis of functional improvement in response to human umbilical cord blood stem cell transplantation in hearts with postinfarct LV remodeling. Cell Transplantation 24(6):971-983.

Cui L, Jia X, Zhou Q, Zhai X, Zhou Y, Zhu H (2014). Curcumin affects $\beta$-catenin pathway in hepatic stellate cell in vitro and in vivo. Journal of Pharmacy and Pharmacology 66(11):1615-1622.

D’Argenio G, Mazzone G, Ribecco MT, Lembo V, Vitaglione P, Guarino M, Morisco F, Napolitano M, Fogliano V, Caporaso N (2013). Garlic extract attenuating rat liver fibrosis by inhibiting TGF- $\beta 1$. Clinical Nutrition 32(2):252-258.

Dong H, Lu Fe, Zhao $\mathbf{L}$ (2012). Chinese herbal medicine in the treatment of nonalcoholic fatty liver disease. Chinese Journal of Integrative Medicine 18(2):152-160.

Duval F, Moreno-Cuevas JE, González-Garza MT, Maldonado-Bernal C, CruzVega DE (2015). Liver fibrosis and mechanisms of the protective action of medicinal plants targeting inflammation and the immune response. International Journal of Inflammation 2015:14 pages.

Duval F, Moreno-Cuevas JE, González-Garza MT, Rodríguez-Montalvo C, CruzVega DE (2014). Liver fibrosis and protection mechanisms action of medicinal plants targeting apoptosis of hepatocytes and hepatic stellate cells. Advances in Pharmacological Sciences 2014:11 pages.

El AE-DE-S, Sokar SS, Shebl AM, Mohamed DZ (2017). Antifibrotic effect of diethylcarbamazine combined with hesperidin against ethanol induced liver fibrosis in rats. Biomedicine \& Pharmacotherapy 89:1196-1206.

Elmahdy NA, Sokar SS, Salem ML, Sarhan NI, Abou-Elela SH (2017). Anti-fibrotic potential of human umbilical cord mononuclear cells and mouse bone marrow cells in CCl4-induced liver fibrosis in mice. Biomedicine \& Pharmacotherapy 89:1378-1386.

Elsharkawy A, Oakley F, Mann D (2005). The role and regulation of hepatic stellate cell apoptosis in reversal of liver fibrosis. Apoptosis 10(5):927-939.

Elshazly SM, Mahmoud AA (2014). Antifibrotic activity of hesperidin against dimethylnitrosamine-induced liver fibrosis in rats. Naunyn-Schmiedeberg's Archives of Pharmacology 387(6):559-567.

Farkhondeh T, Samarghandian S, Azimin-Nezhad M, Samini F (2015). Effect of chrysin on nociception in formalin test and serum levels of noradrenalin and corticosterone in rats. International Journal of Clinical and Experimental Medicine 8(2):2465-2470. 
Ferry DR, Smith A, Malkhandi J, Fyfe DW, deTakats PG, Anderson D, Baker J, Kerr DJ (1996). Phase I clinical trial of the flavonoid quercetin: pharmacokinetics and evidence for in vivo tyrosine kinase inhibition. Clinical Cancer Research 2(4):659-668.

Gedik N, Kabasakal L, Şehirli Ö, Ercan F, Sirvanci S, Keyer-Uysal M, Sener G (2005). Long-term administration of aqueous garlic extract (AGE) alleviates liver fibrosis and oxidative damage induced by biliary obstruction in rats. Life Sciences 76(22):2593-2606.

Gressner OA, Gressner AM (2008). Connective tissue growth factor: a fibrogenic master switch in fibrotic liver diseases. Liver International 28(8):1065-1079.

Ha H-L, Shin H-J, Feitelson MA, Yu D-Y (2010). Oxidative stress and antioxidants in hepatic pathogenesis. World Journal of Gastroenterology: WJG 16(48):60356043.

Hammel P, Couvelard A, O'Toole D, Ratouis A, Sauvanet A, Fléjou JF, Degott C, Belghiti J, Bernades P, Valla D, Ruszniewski P, Lévy P (2001). Regression of liver fibrosis after biliary drainage in patients with chronic pancreatitis and stenosis of the common bile duct. New England Journal of Medicine 344(6):418-423.

Harwood M, Danielewska-Nikiel B, Borzelleca J, Flamm G, Williams G, Lines T (2007). A critical review of the data related to the safety of quercetin and lack of evidence of in vivo toxicity, including lack of genotoxic/carcinogenic properties. Food and Chemical Toxicology 45(11):2179-2205.

He J, Giusti MM (2010). Anthocyanins: natural colorants with health-promoting properties. Annual Review of Food Science and Technology 1:163-187.

Huang SS, Chen Dz, Wu H, Chen RC, Du SJ, Dong JJ, Liang G, Xu LM, Wang XD, Yang YP, Yu ZP, Feng WK, Chen YP. (2016). Cannabinoid receptors are involved in the protective effect of a novel curcumin derivative C66 against CCl4-induced liver fibrosis. European Journal of Pharmacology 779:22-30.

Inagaki Y, Okazaki I (2007). Emerging insights into transforming growth factor $\beta$ Smad signal in hepatic fibrogenesis. Gut 56(2):284-292.

Jeong DH, Lee G-P, Jeong W-I, Do SH, Yang HJ, Yuan DW, Park HY, Kim KJ, Jeong KS (2005). Alterations of mast cells and TGF- $\beta 1$ on the silymarin treatment for CCl4-induced hepatic fibrosis. World Journal of Gastroenterology 11(8):1141-1148.

Jiang Y, Jahagirdar BN, Reinhardt RL, Schwartz RE, Keene CD, Ortiz-Gonzalez XR, Reyes M, Lenvik T, Lund T, Blackstad M, Du J, Aldrich S, Lisberg A, Low WC, Largaespada DA, Verfaillie CM (2002). Pluripotency of mesenchymal stem cells derived from adult marrow. Nature 418(6893):41-49.

Jing J, Zhao J, Hua B, Xue MQ, Zhu YF, Liu G, Wang YH, Peng XD (2015). Inhibitory effect of flavonoids from Glycyrrhiza uralensis on expressions of TGF- $\beta 1$ and Caspase-3 in thioacetamide-induced hepatic fibrosis in rats. China Journal of Chinese Materia Medica 40(15):3034-3040.

Jung KH, Shin HP, Lee S, Lim YJ, Hwang SH, Han H, Park HK, Chung JH, Yim SV (2009). Effect of human umbilical cord blood-derived mesenchymal stem cells in a cirrhotic rat model. Liver International 29(6):898-909.

Kawada N (2011). Evolution of hepatic fibrosis research. Hepatology Research 41(3):199-208. 
Kessoku T, Imajo K, Honda Y, Kessoku T, Imajo K, Honda Y, Kato T, Ogawa Y, Tomeno, Kato S, Mawatari H, Fujita K, Yoneda M, Nagashima Y, Saito S, Wada K, Nakajima A (2016). Resveratrol ameliorates fibrosis and inflammation in a mouse model of nonalcoholic steatohepatitis. Scientific Reports 6:22251-22261.

Kisseleva T, Brenner DA (2008). Mechanisms of fibrogenesis. Experimental Biology and Medicine 233(2):109-122.

Knittel T, Mehde M, Kobold D, Saile B, Dinter C, Ramadori G (1999). Expression patterns of matrix metalloproteinases and their inhibitors in parenchymal and non-parenchymal cells of rat liver: regulation by TNF- $\alpha$ and TGF- $\beta 1$. Journal of Hepatology 30(1):48-60.

Lang A, Schrum LW, Schoonhoven R, Tuvia S, Solís-Herruzo JA, Tsukamoto H, Brenner DA, Rippe RA (2000). Expression of small heat shock protein $\alpha B$ crystallin is induced after hepatic stellate cell activation. American Journal of Physiology-Gastrointestinal and Liver Physiology 279(6):G1333-G1342.

Latief U, Ahmad R (2018). Herbal remedies for liver fibrosis: A review on the mode of action of fifty herbs. Journal of Traditional and Complementary Medicine 8(3):352-360.

Lee J-H, Jang EJ, Seo HL, Ku SK, Lee JR, Shin SS, Park SD, Kim SC, Kim YW (2014). Sauchinone attenuates liver fibrosis and hepatic stellate cell activation through TGF- $\beta /$ Smad signaling pathway. Chemico-Biological Interactions 224:58-67.

Li Q, Zhou X, Shi Y, Li J, Zheng L, Cui L, Zhang J, Wang L, Han Z, Han Y, Fan D (2013). In vivo tracking and comparison of the therapeutic effects of MSCs and HSCs for liver injury. PloS One 8(4):e62363-62373.

Li X, Peng X, Zhang W, Dai L (2008). Inhibiting effects of denshensu, baicalin, astragalus and Panax notoginseng saponins on hepatic fibrosis and their possible mechanisms. Chinese Journal of Hepatology 16(3):193-197.

Liang B, Guo X-L, Jin J, Ma Y-C, Feng Z-Q (2015). Glycyrrhizic acid inhibits apoptosis and fibrosis in carbon-tetrachloride-induced rat liver injury. World Journal of Gastroenterology 21(17):5271-5280.

Lim Y-S, Kim WR (2008). The global impact of hepatic fibrosis and end-stage liver disease. Clinics in Liver Disease 12(4):733-746.

Lin H, Xu R, Zhang Z, Chen L, Shi M, Wang F-S (2011). Implications of the immunoregulatory functions of mesenchymal stem cells in the treatment of human liver diseases. Cellular \& Molecular Immunology 8(1):19-22.

Liu CP, Tsai WJ, Shen CC, Lin YL, Liao JF, Chen CF, Kuo YC (2006). Inhibition of (S)-armepavine from Nelumbo nucifera on autoimmune disease of MRL/MpJ-lpr/lpr mice. European Journal of Pharmacology 531(1-3):270279.

Lu B, Yin L, Xu L, Peng J (2011). Application of proteomic and bioinformatic techniques for studying the hepatoprotective effect of dioscin against $\mathrm{CCl} 4$ induced liver damage in mice. Planta Medica 77(05):407-415.

Mahmoud MF, Zakaria S, Fahmy A (2014). Aqueous garlic extract alleviates liver fibrosis and renal dysfunction in bile-duct-ligated rats. Zeitschrift für Naturforschung C 69(3-4):133-141.

Maizels R, Denham D (1992). Diethylcarbamazine (DEC): immunopharmacological interactions of an anti-filarial drug. Parasitology 105(S1):S49-S60. 
Mormone E, George J, Nieto N (2011). Molecular pathogenesis of hepatic fibrosis and current therapeutic approaches. Chemico-Biological Interactions 193(3):225231.

Nasir GA, Mohsin S, Khan M, Shams S, Ali G, Khan SN, Riazuddin S (2013). Mesenchymal stem cells and Interleukin-6 attenuate liver fibrosis in mice. Journal of Translational Medicine 11(1):78-86.

Oertel M, Shafritz DA (2008). Stem cells, cell transplantation and liver repopulation. Biochimica et Biophysica Acta (BBA)-Molecular Basis of Disease 1782(2):61-74.

Peng XD, Dai LL, Huang CQ, He CM, Chen LJ (2009). Correlation between antifibrotic effect of baicalin and serum cytokines in rat hepatic fibrosis. World Journal of Gastroenterology 15(37):4720-4725.

Pérez-Vargas JE, Zarco N, Shibayama M, Segovia J, Tsutsumi V, Muriel P (2014). Hesperidin Prevents Liver Fibrosis in Rats by Decreasing the Expression of Nuclear Factor- $\kappa B$, Transforming Growth Factor $-\beta$ and Connective Tissue Growth Factor. Pharmacology 94(1-2):80-89.

Pinzani M, Gesualdo L, Sabbah GM, Abboud H (1989). Effects of platelet-derived growth factor and other polypeptide mitogens on DNA synthesis and growth of cultured rat liver fat-storing cells. The Journal of Clinical Investigation 84(6):1786-1793.

Poynard T, Mathurin P, Lai CL, Guyader D, Poupon R, Tainturier MH, Myers RP, Muntenau M, Ratziu V, Manns M, Vogel A, Capron F, Chedid A, Bedossa $P$ (2003). A comparison of fibrosis progression in chronic liver diseases. Journal of Hepatology 38(3):257-265.

Qiu J, Zhou Q, Zhai X, Jia X, Zhou Y (2014). Curcumin regulates delta-like homolog 1 expression in activated hepatic stellate cell. European Journal of Pharmacology 728:9-15.

Qu Y, Zong L, Xu M, Dong Y, Lu L (2015). Effects of 18 $\alpha$-glycyrrhizin on TGF$\beta 1 /$ Smad signaling pathway in rats with carbon tetrachloride-induced liver fibrosis. International Journal of Clinical and Experimental Pathology 8(2):1292-1301.

Rabani V, Shahsavani M, Gharavi M, Piryaei A, Azhdari Z, Baharvand H (2010). Mesenchymal stem cell infusion therapy in a carbon tetrachloride-induced liver fibrosis model affects matrix metalloproteinase expression. Cell Biology International 34(6):601-605.

Ramon B, David A, Brenner D (2005). Liver fibrosis. J Clin Invest 115(2):209-218.

Reeves HL, Friedman SL (2002). Activation of hepatic stellate cells-a key issue in liver fibrosis. Front Biosci 7(4):808-826.

Rocha SWS, de França ME, Rodrigues GB, Barbosa KP, Nunes AK, Pastor AF, Oliveira AG, Oliveira WH, Luna RL, Peixoto CA (2014). Diethylcarbamazine reduces chronic inflammation and fibrosis in carbon tetrachloride-(CCl4-) induced liver injury in mice. Mediators of Inflammation 2014:15 pages.

Rocha SWS, Silva BS, Gomes FO, Silva AK, Raposo C, Barbosa KP, Dde O, dosSantos AC, Peixoto CA (2012). Effect of diethylcarbamazine on chronic hepatic inflammation induced by alcohol in C57BL/6 mice. European Journal of Pharmacology 689(1-3):194-203. 
Schuppan D, Ruehl M, Somasundaram R, Hahn EG. (2001). Matrix as a modulator of hepatic fibrogenesis. In: Seminars in liver disease,. vol 21. by Thieme Medical Publishers,New York pp 351-372.

Seki E, Schwabe RF (2015). Hepatic inflammation and fibrosis: functional links and key pathways. Hepatology 61(3):1066-1079.

Shiota G, Kunisada T, Oyama K, Udagawa A, Nomi T, Tanaka K, Tsutsumi A, Isono M, Nakamura T, Hamada H, Sakatani T, Sell S, Sato K, Ito H, Kawasaki H (2000). In vivo transfer of hepatocyte growth factor gene accelerates proliferation of hepatic oval cells in a 2acetylaminofluorene/partial hepatectomy model in rats. FEBS Letters 470(3):325-330.

Sidhu P, Shankargouda S, Rath A, Ramamurthy PH, Fernandes B, Singh AK (2018). Therapeutic benefits of liquorice in dentistry. Journal of Ayurveda and Integrative Medicine.

Sokar SS, El-Sayad ME-S, Ghoneim ME-S, Shebl AM (2017). Combination of Sitagliptin and Silymarin ameliorates liver fibrosis induced by carbon tetrachloride in rats. Biomedicine \& Pharmacotherapy 89:98-107.

Subrahmanyam D (1987). Antifilarials and their mode of action. Filariasis 127:246264.

Sudo K, Yamada Y, Saito K, Shimizu S, Ohashi H, Kato T, Moriwaki H, Ito H, Seishima M (2008). TNF- $\alpha$ and IL-6 signals from the bone marrow derived cells are necessary for normal murine liver regeneration. Biochimica et Biophysica Acta (BBA)-Molecular Basis of Disease 1782(11):671-679.

Sugihara A, Tsujimura T, Fujita Y, Nakata Y, Terada N (1999). Evaluation of role of mast cells in the development of liver fibrosis using mast cell-deficient rats and mice. Journal of Hepatology 30(5):859-867.

Sun J, Wu Y, Long C, He P, Gu J, Yang L, Liang Y, Wang Y (2018). Anthocyanins isolated from blueberry ameliorates $\mathrm{CCl} 4$ induced liver fibrosis by modulation of oxidative stress, inflammation and stellate cell activation in mice. Food and Chemical Toxicology 120:491-499.

Sung SH, Kim YC (2000). Hepatoprotective diastereomeric lignans from Saururus chinensis herbs. Journal of Natural Products 63(7):1019-1021.

Tanabe Y, Tajima F, Nakamura Y, Shibasaki E, Wakejima M, Shimomura T, Murai R, Murawaki Y, Hashiguchi K, Kanbe T, Saeki T, Ichiba M, Yoshida Y, Mitsunari M, Yoshida S, Miake J, Yamamoto Y, Nagata N, Harada T, Kurimasa A, Hisatome I, Terakawa N, Murawaki Y, Shiota G (2004). Analyses to clarify rich fractions in hepatic progenitor cells from human umbilical cord blood and cell fusion. Biochemical and Biophysical Research Communications 324(2):711-718.

Tang Y (2015). Curcumin targets multiple pathways to halt hepatic stellate cell activation: updated mechanisms in vitro and in vivo. Digestive Diseases and Sciences 60(6):1554-1564.

Tsai JH, Liu JY, Wu TT, Ho PC, Huang CY, Shyu JC, Hsieh YS, Tsai CC, Liu YC (2008). Effects of silymarin on the resolution of liver fibrosis induced by carbon tetrachloride in rats. Journal of Viral Hepatitis 15(7):508-514.

Vestergaard $M$, Ingmer $\mathbf{H}$ (2019). Antibacterial and antifungal properties of resveratrol. International Journal of Antimicrobial Agents 53:716-723. 
Wan J, Benkdane M, Teixeira- Clerc F, Bonnafous S, Louvet A, Lafdil F, Pecker F, Tran A, Gual P, Mallat A, Lotersztajn S, Pavoine C (2014). M2 Kupffer cells promote M1 Kupffer cell apoptosis: a protective mechanism against alcoholic and nonalcoholic fatty liver disease. Hepatology 59(1):130142.

Wang C, Duan X, Sun X, Liu Z, Sun P, Yang X, Sun H, Liu K, Meng Q (2016). Protective effects of glycyrrhizic acid from edible botanical glycyrrhiza glabra against non-alcoholic steatohepatitis in mice. Food \& Function 7(9):3716-3723.

Wang R, Zhang H, Wang Y, Song F, Yuan Y (2017). Inhibitory effects of quercetin on the progression of liver fibrosis through the regulation of NF-кB/ІкB $\alpha$, p38 MAPK, and Bcl-2/Bax signaling. International Immunopharmacology 47:126-133.

Wang Y, Guan M, Zhao X, Li X (2018). Effects of garlic polysaccharide on alcoholic liver fibrosis and intestinal microflora in mice. Pharmaceutical Biology 56(1):325-332.

Weiler-Normann C, Herkel J, Lohse A (2007). Mouse models of liver fibrosis. Zeitschrift für Gastroenterologie 45(01):43-50.

Weiss A, Attisano L (2013). The TGFbeta superfamily signaling pathway. Wiley Interdisciplinary Reviews: Developmental Biology 2(1):47-63.

Weng TC, Shen CC, Chiu YT, Lin YL, Kuo CD, Huang YT (2009). Inhibitory effects of armepavine against hepatic fibrosis in rats. Journal of Biomedical Science 16(1):78-90.

Weng TC, Shen CC, Chiu YT, Lin YL, Huang YT (2012). Effects of armepavine against hepatic fibrosis induced by thioacetamide in rats. Phytotherapy Research 26(3):344-353.

WHO Organization (2005). Revised global burden of disease (GBD) 2002 estimates. Genebra: Horld Health Organization. http://www.who.int/healthinfo/bodgbd2002revised/en/index.html

Wu L, Zhang Q, Mo W, Feng J, Li S, Li J, Liu T, Xu S, Wang W, Lu X, Yu Q, Chen K, Xia Y, Lu J, Xu L, Zhou Y, Fan X, Guo C (2017). Quercetin prevents hepatic fibrosis by inhibiting hepatic stellate cell activation and reducing autophagy via the TGF- $\beta 1 /$ Smads and PI3K/Akt pathways. Scientific Reports 7(1):9289-9301.

Xu R, Zhang Z, Wang FS (2012). Liver fibrosis: mechanisms of immune-mediated liver injury. Cellular \& Molecular Immunology 9(4):296-301.

Yao Qy, Xu Bl, Wang Jy, Liu Hc, Zhang Sc, Tu Ct (2012). Inhibition by curcumin of multiple sites of the transforming growth factor-betal signalling pathway ameliorates the progression of liver fibrosis induced by carbon tetrachloride in rats. BMC Complementary and Alternative Medicine 12(1):156-166.

Yoshida K, Matsuzaki K (2012). Differential regulation of TGF- $\beta /$ Smad signaling in hepatic stellate cells between acute and chronic liver injuries. Frontiers in Physiology 3:53-59.

Yu F, Ji S, Su L, Wan L, Zhang S, Dai C, Wang Y, Fu J, Zhang Q (2015). Adiposederived mesenchymal stem cells inhibit activation of hepatic stellate cells in vitro and ameliorate rat liver fibrosis in vivo. Journal of the Formosan Medical Association 114(2):130-138. 
Zeng Y, Song C, Ding X, Ji X, Yi L, Zhu K (2007). Baicalin reduces the severity of experimental autoimmune encephalomyelitis. Brazilian Journal of Medical and Biological Research 40(7):1003-1010.

Zhang F, Zhang Z, Chen L, Kong D, Zhang X, Lu C, Lu Y, Zheng S (2014). Curcumin attenuates angiogenesis in liver fibrosis and inhibits angiogenic properties of hepatic stellate cells. Journal of Cellular and Molecular Medicine 18(7):1392-1406.

Zhang X, Han X, Yin L, Xu L, Qi Y, Xu Y, Sun H, Lin Y, Liu K, Peng J (2015a). Potent effects of dioscin against liver fibrosis. Scientific Reports 5:97139723.

Zhang X, Xu Y, Qi Y, Han X, Yin L, Xu L, Liu K, Peng J (2015b). Potent effects of dioscin against thioacetamide-induced liver fibrosis through attenuating oxidative stress in turn inhibiting inflammation, TGF- $\beta / \mathrm{Smad}$ and MAPK signaling pathways. Journal of Functional Foods 16:436-447.

Zhou P, Hohm S, Olusanya Y, Hess DA, Nolta J (2009). Human progenitor cells with high aldehyde dehydrogenase activity efficiently engraft into damaged liver in a novel model. Hepatology 49(6):1992-2000. 
استخدام المركبات المشتقه طبيعياً فى الوقايه أوالحد من التليف الكبدى المحدث تجريبياً

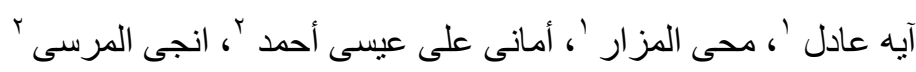

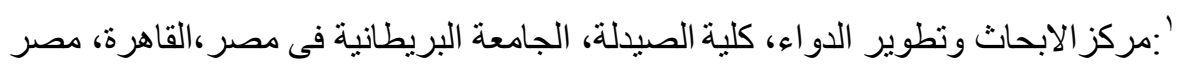

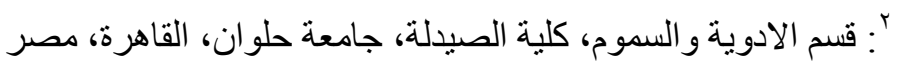

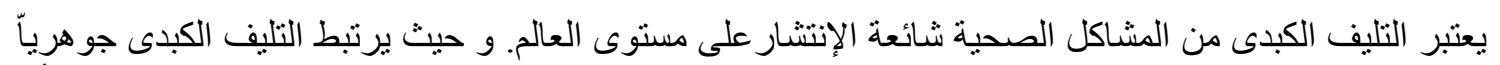

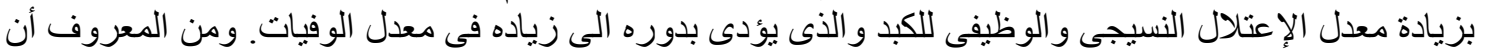

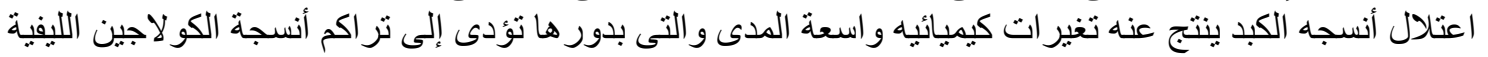

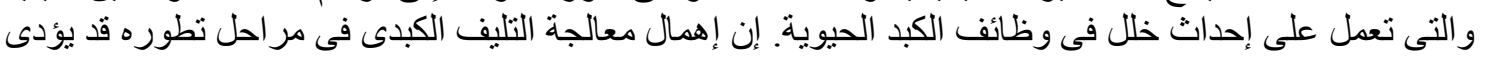

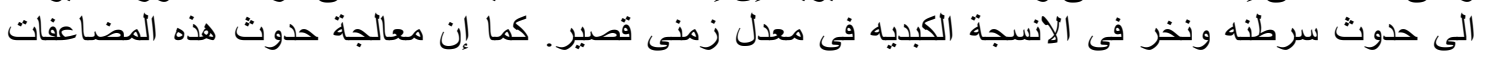

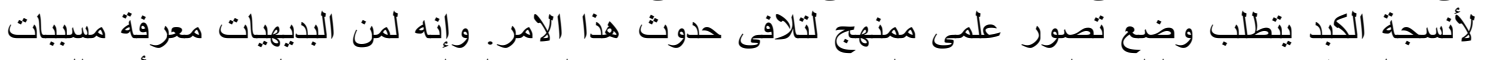

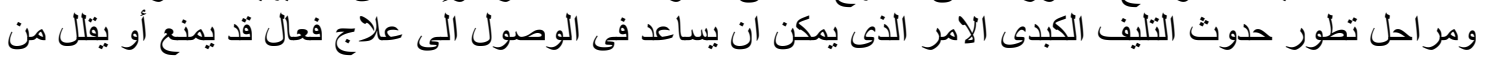
مر احل إحداثه.

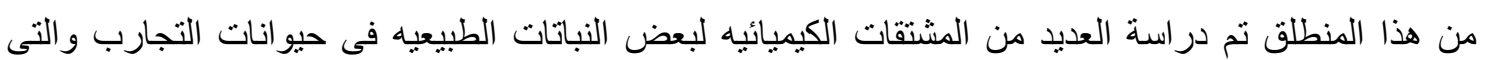

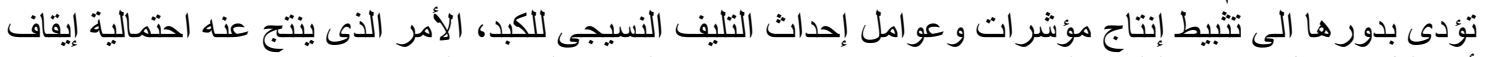

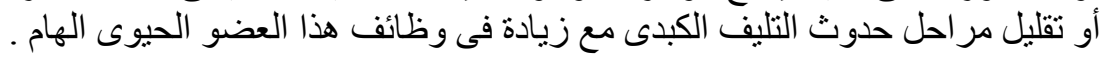

